\title{
Bitcoin and global financial stress: a copula-based approach to dependence and causality in the quantiles
}

Article

Accepted Version

Creative Commons: Attribution-Noncommercial-No Derivative Works 4.0

Bouri, E., Gupta, R., Lau, C. K. M., Roubaud, D. and Wang, S. (2018) Bitcoin and global financial stress: a copula-based approach to dependence and causality in the quantiles. The Quarterly Review of Economics and Finance, 69. pp. 297-307. ISSN 1062-9769 doi:

https://doi.org/10.1016/j.qref.2018.04.003 Available at https://centaur.reading.ac.uk/79660/

It is advisable to refer to the publisher's version if you intend to cite from the work. See Guidance on citing.

To link to this article DOI: http://dx.doi.org/10.1016/j.qref.2018.04.003

Publisher: Elsevier

All outputs in CentAUR are protected by Intellectual Property Rights law, including copyright law. Copyright and IPR is retained by the creators or other copyright holders. Terms and conditions for use of this material are defined in the End User Agreement. 


\section{CentAUR}

Central Archive at the University of Reading

Reading's research outputs online 


\section{Accepted Manuscript}

Title: Bitcoin and global financial stress: A copula-based approach to dependence and causality in the quantiles

Authors: Elie Bouri, Rangan Gupta, Chi Keung Marco Lau, David Roubaud, Shixuan Wang

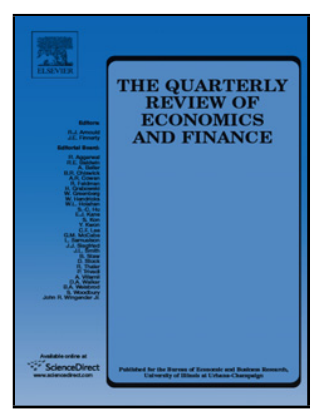

PII:

S1062-9769(17)30324-1

DOI: https://doi.org/10.1016/j.qref.2018.04.003

Reference: QUAECO 1123

To appear in: The Quarterly Review of Economics and Finance

Received date: $\quad 7-10-2017$

Revised date: $\quad$ 4-3-2018

Accepted date: $\quad 3-4-2018$

Please cite this article as: Bouri E, Gupta R, Lau CKM, Roubaud D, Wang S, Bitcoin and global financial stress: A copula-based approach to dependence and causality in the quantiles, Quarterly Review of Economics and Finance (2010), https://doi.org/10.1016/j.qref.2018.04.003

This is a PDF file of an unedited manuscript that has been accepted for publication. As a service to our customers we are providing this early version of the manuscript. The manuscript will undergo copyediting, typesetting, and review of the resulting proof before it is published in its final form. Please note that during the production process errors may be discovered which could affect the content, and all legal disclaimers that apply to the journal pertain. 


\section{Bitcoin and global financial stress: A copula-based approach to dependence and causality in the quantiles}

Elie Bouri ${ }^{\mathrm{a}}$, Rangan Gupta ${ }^{\mathrm{b}}$, Chi Keung Marco Lau ${ }^{\mathrm{c}}$, David Roubaud ${ }^{\mathrm{d}}$, Shixuan Wang ${ }^{\mathrm{e}}$

${ }^{a}$ USEK Business School, Holy Spirit University of Kaslik, Jounieh, Lebanon, Email: eliebouri@usek.edu.lb.

${ }^{b}$ Department of Economics, University of Pretoria, Pretoria, 0002, South Africa, Email: rangan.gupta@up.ac.za.

${ }^{\mathrm{c}}$ Department of Accountancy, Finance and Economics, Huddersfield Business School, University of Huddersfield, Queensgate, Huddersfield, HD1 3DH, UK. Email: c.lau@ hud.ac.uk

${ }^{\mathrm{d}}$ Energy and Sustainable Development (ESD), Montpellier Business School, Montpellier, France, Email:d.roubaud@montpellier-bs.com.

${ }^{\text {e}}$ Cardiff Business School, Cardiff University, CF10 3EU, United Kingdom, Email: wsx423104361@gmail.com.

\section{Corresponding author:}

Elie Bouri

USEK Business School, Holy Spirit University of Kaslik, Jounieh, Lebanon. POBOX 446, Jounieh, Lebanon. Phone: +961900861. Email: eliebouri@usek.edu.lb.

\section{Highlights}

- Study daily dependence between global financial stress and Bitcoin

- Use various forms of copula models: standard and quantiles-based

- Financial stress causes Bitcoin returns at left and right tail of the latter's conditional distribution

- Financial stress, however, has limited directional predictability for Bitcoin 


\begin{abstract}
We apply different techniques and uncover the quantile conditional dependence between the global financial stress index and Bitcoin returns from July 18, 2010, to December 29, 2017. The results from the copula-based dependence show evidence of right-tail dependence between the global financial stress index and Bitcoin returns. We focus on the conditional quantile dependence and indicate that the global financial stress index strongly Granger-causes Bitcoin returns at the left and right tail of the distribution of the Bitcoin returns, conditional on the global financial stress index. Finally, we use a bivariate cross-quantilogram approach and show only limited directional predictability from the global financial stress index to Bitcoin returns in the medium term, for which Bitcoin can act as a safe-haven against global financial stress.
\end{abstract}

Keywords: Bitcoin; global financial stress index; dependence; copula; quantiles

JEL classification: C22, G15

\title{
1. Introduction
}

Bitcoin was first designed in 2009 to allow users to send and receive payments on a peer-to-peer basis. However, its popularity as an investment asset has considerably increased as speculators and investors store Bitcoin with the objective of increasing its scarcity and potentially driving increases in its value. From July 2010 to December 2018, Bitcoin has quickly increased from less than one USD to more than fourteen thousand US dollars ${ }^{1}$. Importantly, Bitcoin has shown some resilience during periods of stress (Weber, 2014; Bouri et al., 2017a; Luther and Salter, 2017).

Prior causality studies suggest that Bitcoin might serve as a hedge against equities and currencies (Bouri et al., 2017b, Dyhrberg, 2016b), the commodity index (Bouri et al., 2017b,), and stock market's expectation of near term volatility - as measured by the VIX (Bouri et al. (2017a).

\footnotetext{
${ }^{1}$ At the end of December 2018, Bitcoin's market value reached 216.32 billion US dollars, based on a closing price of 14,156 US dollars (https://coinmarketcap.com/currencies/bitcoin/).
} 
Nonetheless, the relationship between Bitcoin and a global measure of financial stress has remained unexplored. To enrich the related empirical literature, this study examines the relationship between Bitcoin returns and global financial stress index.

On the data level, this study considers the global financial stress index (GFSI) that was recently introduced by Bank of America Merrill Lynch. The GFSI captures better global stress than the VIX measure ${ }^{2}$ employed by Bouri et al. (2017b). Specifically, the GFSI aggregates 23 measures of stress covering three types of financial market stress (risk, hedging demand, and investor appetite for risk) across five asset classes (credit, equity, interest rates, forex and commodity markets) and various geographies. On the methodological level, this study employs a combination between copula function and quantile causality, which allows for uncovering the Granger causality in both distributions and quantiles. As such, practitioners, scholars and policymakers would extend their limited understanding of the ability of Bitcoin to act as a safe-haven against global financial stress.

Empirical analyses show evidence of right-tail dependence between the GFSI and Bitcoin returns, and further show that the GSFI strongly Granger-causes Bitcoin returns at the left tail (i.e., during deficient performance) and the right tail (i.e. during very robust performance) but not at the middle (i.e., during average performance) of the distribution of the Bitcoin returns conditional on the GSFI. Further analysis indicates that Bitcoin can act as a safe-haven against global financial stress from a medium-term perspective.

\section{Research background}

Bitcoin is an innovative peer-to-peer electronic payment network that uses a cryptography protocol to secure transactions. The building block of the network relies on an underlying blockchain technology that records and secures all Bitcoin transactions. Blockchain is a distributed ledger made of an unchangeable chain of data blocks spread across multiple sites but chained together cryptographically.

Bitcoin operates in the absence of a central authority. Specifically, trust in Bitcoin is distributed to a large network and established through mass collaboration without a powerful third party. Unlike the case of conventional fiat currencies, Bitcoin production is neither centralized nor

\footnotetext{
${ }^{2}$ http://uk.reuters.com/article/markets-stressindex-idUSN2920764420101129.
} 
subject to inflation (Ciaian and Rajcaniova, 2016). Instead, it is dictated by the protocol that limits the number of Bitcoin in circulation to 21 million. While Bitcoin can be produced as a reward for approving Bitcoin transactions in a process called "mining", it can be bought and sold against conventional currencies on trading platforms or exchanges.

Bitcoin has shown some resilience during periods of stress, suggesting a potential hedging ability. Weber (2014) and Bouri et al. (2017a) argue that the global uncertainty that accompanied and followed the 2008 global financial crisis facilitated the rapid emergence of Bitcoin as both a financial asset and an alternative currency to conventional economies. Importantly, later stress periods such as the European sovereign debt crisis of 2010-2013 and the Cypriot banking crisis of 2012-2013 have further driven the use of Bitcoin as a shelter from sovereign and systematic risk (Bouri et al., 2017a). Luther and Salter (2017) show that interest in Bitcoin substantially increased following the March 16, 2013, announcement that Cyprus would accept a bailout. Increasing interest in Bitcoin has also been reported in countries such as Greece and Spain, whose banks are troubled. Bitcoin is an alternative to sovereign currencies and is often considered part of an alternative economy. In an environment of high uncertainty and low trust, investors move away from main-state economies and often resort to Bitcoin (Bouri et al., 2017b). Bitcoin has been referred to as digital gold (Popper, 2015), and Dyhrberg (2016a) situates its hedging capability somewhere between gold and the US dollar.

Several empirical studies have noted the valuable role of Bitcoin as an investment and have highlighted the diversification benefits of adding Bitcoin to an equity portfolio. Brière et al. (2015) use weekly data from 2010 to 2013 and highlight the low correlation of Bitcoin with both traditional assets (worldwide stocks, bonds, and hard currencies) and alternative investments (commodities, hedge funds, and real estate). The authors note Bitcoin's significant diversification benefits despite its extremely high average return and volatility. Dyhrberg (2016b) shows that Bitcoin is useful as a hedge for UK currency and equities. Bouri et al. (2017b) indicate that Bitcoin can serve as an effective diversifier for major world stock indices, bonds, oil, gold, the general commodity index and the US dollar index. Those authors also reveal that Bitcoin has hedging and safe-haven properties against Asian Pacific and Chinese stocks. Ji et al. (2017) show that Bitcoin is isolated from the conventional global financial system. Bouri et al. (2017c) note the safe-haven property of Bitcoin against equities and reveal that Bitcoin is negatively related to the US VIX. 
The empirical relationship between Bitcoin and global financial stress has been largely ignored. To the best of our knowledge, the only work closely related to this study was conducted by Bouri et al. (2017a), who examine Bitcoin's hedging ability against the VIXs of developed and emerging markets. After decomposing Bitcoin returns into different frequencies and applying quantile-on-quantile regressions, the authors show that Bitcoin does act as a hedge against global uncertainty at both the lower and the upper ends of Bitcoin returns and global uncertainty, particularly on shorter investment horizons. Although Bouri et al. (2017a) differentiate between short and long investment horizons and between upper and lower quantiles, they ignore the dependence structure, as captured by copula, along with Granger-causality in both distributions and quantiles. Furthermore, Bank of America Merrill Lynch argues that the breadth and depth of the GFSI make it a more accurate gauge of global stress than the VIX, which is based on option data. More specifically, the GFSI helps detect significant market turning points, as indicated by the back-testing that has shown the high degree of the GFSI's accuracy in forecasting market sell-offs since 2000. Based on the above, it appears that the GFSI is an essential tool for market participants to make better investment and risk management decisions. Importantly, the increased knowledge of which risks are essential and against which to hedge them in the different quantiles whilst explaining the copula dependence structure are two crucial aspects of successful investing. It follows that the hedging property of Bitcoin against that global measure of financial stress requires a thorough examination via the copula function and the Granger causality in both distribution and quantiles. This is where this contributes to the related literature.

\section{Data and methods}

\subsection{Data}

Data used in this study are daily (5 days per week) and cover the period from July 18, 2010, to December 29, 2017. It consist of Bitcoin prices and the GFSI. Bitcoin prices are collected from CoinDesk (www.coindesk.com/price) and represent the average price of Bitcoin across leading exchanges (Bouri et al., 2017a). Data on the GFSI are collected from the Bloomberg Terminal. The GFSI is an index for global financial distress that better captures global stress than the VIX. Introduced in November 2010 by Bank of America Merrill Lynch, the GFSI aggregates 23 measures of stress covering three types of financial market stress (risk, hedging demand, and 
investor appetite for risk) across five asset classes (credit, equity, interest rates, forex and commodity markets) and various geographies. Levels greater than zero indicate more financial market stress than normal, and vice-versa. Table 1 shows the summary statistics of the GFSI and the return on Bitcoin (RBC) (calculated as the difference in logarithm between two consecutive prices). There are several noteworthy observations. While both the GFSI and RBC have positive means, GFSI has a larger standard deviation. The GFSI and RBC are positively skewed. RBC has significant excess kurtosis, whereas the GFSI has modest kurtosis. The Jarque-Bera test shows that the GFSI and RBC are not following a normal distribution. The GFSI and RBC are negative correlated $(-0.053)$.

\subsection{Methods}

The empirical analyses rely on three main approaches to uncover the quantile conditional dependence between GFSI and Bitcoin returns (RBC). The first one is the dependence via copulas, which can characterize the average movements and the joint extreme movements between the two examined variables. The second approach is the out-of-sample approach of Hong and Li (2005), called the Granger causality in distribution (GCD), which captures the Granger causality in distributions in each conditional quantile. The third one is the crossquantilogram approach of Han et al. (2016), which allows to measure of directional predictability in quantiles.

\subsubsection{Modelling dependence using copulas}

It is well documented that copula functions provide both flexibility and effectiveness in characterizing such movement patterns, allowing obtaining valuable information on the average dependence and tail dependence.

A copula is a multivariate cumulative distribution function, and its marginal distributions are uniform on the interval $[0,1]$. Nelsen (1999) review the rigorous mathematical foundation of copulas. Sklar's theorem plays the central role in the theory of copulas. "Sklar's theorem elucidates the role that copulas play in the relationship between multivariate distribution functions and their univariate margin" (Nelsen, 1999) ${ }^{3}$.

\footnotetext{
${ }^{3}$ Details on the Copula dependence model and the Sklar's theorem are given in the Appendix.
} 
There are at least three advantages of using copulas in analyzing the dependence. First, the copula method is designed to capture the complex and non-linear dependence structure of a multivariate distribution, whilst the traditional Pearson correlation assumes a linear dependence relationship and is not capable of measuring the asymmetric dependence. Copulas enable us to find both the tail dependence and the asymmetric dependence. Importantly, the tail dependence can measure the probability of simultaneous extreme losses for investors. Second, the marginal behaviour and the dependence structure are separated by the framework of copulas. This separation facilitates both the model specification and the model estimation. Compared with univariate models, the flexibility of the multivariate models is limited. Copulas can jointly combine different univariate models through their copula functions. The estimation can be performed in separate steps for the marginal models and the copula functions. Finally, copulas are invariant to increasing and continuous transformations (Ning, 2010), such as the scaling of logarithm returns, which is commonly used in economic and finance studies.

We apply nine candidate copulas, including normal, Clayton, Rotated Clayton, Plackett, Frank, Gumbel, Rotated Gumbel, Student's t and Symmetrised Joe-Clayton (see the Appendix that includes detailed information on the estimation methods as well as the marginal and Copula models).

Despite their usefulness in modelling the average dependence and tail dependence, copula dependence models do not provide a conclusion about the causality between variables. Thus, we next consider the Granger causality in distribution (GCD) test.

\subsubsection{Granger causality in distribution (GCD) test}

After identifying the appropriateness of adopting copula models and modelling the average dependence and tail dependence, we proceed to uncover the causality dynamic between the GFSI and $\mathrm{RBC}$ by computing the quantile forecasts that rely on the inversion of the parametric conditional copula distribution. We use the model by Lee and Yang (2014) to examine the dependence between the GFSI and RBC using a parametric copula because the linear Granger causality test cannot model the asymmetric dependence between the GFSI and RBC, possibly because of the existence of nonlinearity and structural breaks. From the modelling perspective, it is more informative to explore the causal relationship between the GFSI and RBC using the GCD test, which can model the causal relation at the extremes of the return distributions rather 
than only at the centre. For market practitioners, it is more realistic to imagine that causality is only anticipated at high quantiles of the GFSI and RBC, because RBC may act as a safe haven when the global financial market panics. Indeed, this hypothesis is supported by the empirical evidence we provide later.

We apply the proposed out-of-sample test for GCD in accordance with Hong and Li (2005) with the null hypothesis that $X_{t}$ does not Granger cause $Y_{t}$ in distribution: $H 0: c(u, v)=1$, where $c(u, v)$ is the conditional copula density function, with $\mathrm{u}$ and $\mathrm{v}$ as the conditional probability integral transforms of $\mathrm{X}_{\mathrm{t}}$ (i.e., $\mathrm{GSFI}_{\mathrm{t}}$ ) and $\mathrm{Y}_{\mathrm{t}}$ (i.e., $\mathrm{RBC}_{\mathrm{t}}$ ), respectively. The forecasted conditional variance for $\left\{X_{t}\right\}$ and $\left\{Y_{t}\right\}, \hat{h}_{x, t+1}$ and $\hat{h}_{y, t+1}$, are computed by

$\hat{h}_{x, t+1}=\hat{\beta}_{x 0}+\hat{\beta}_{x 1} x_{t}^{2}+\hat{\beta}_{x 2} \hat{h}_{t, x}$

$\hat{h}_{y, t+1}=\hat{\beta}_{y 0}+\hat{\beta}_{y 1} y_{t}^{2}+\hat{\beta}_{y 2} \hat{h}_{t, x}$

The CDF values of $\hat{u}_{t+1}$ and $\hat{v}_{t+1}$ for $\mathrm{x}_{t+1}$ and $\mathrm{y}_{\mathrm{t}+1}$ are calculated by the empirical distribution function (EDF), and a nonparametric copula function is estimated with pared EDF values $\left\{\hat{u}_{t+1}, \hat{v}_{t+1}\right\}_{t=R}^{T-1}$ using a quartic kernel function specified as follows:

$k(u)=\frac{15}{16}\left(1-u^{2}\right)^{2} I(|u| \leq 1)$

The GCD results using the Hong and $\mathrm{Li}$ (2005) test statistic for $\left\{x_{t+1}, y_{t+1}\right\}_{t=R}^{T-1}$ is 40.843 , which is significant at the $1 \%$ level, indicating that there is significant GCD between the GSFI and RBC. However, evidence of the GCD test does not imply Granger causality in each conditional quantile. In our empirical study, we focus on three regions of the distribution: the left tail (1\% quantile, $5 \%$ quantile and $10 \%$ quantile), the central region (40\% quantile, median and $60 \%$ quantile) and the right tail (90\% quantile, $95 \%$ quantile and $99 \%$ quantile); this is the same as Lee and Yang (2014). The objective is to forecast the conditional quantile, $q_{\alpha}\left(Y_{t} \mid \mathcal{F}_{t}\right)$, where $\alpha$ is the left tail probability. The conditional quantile $q_{\alpha}\left(Y_{t} \mid \mathcal{F}_{t}\right)$ is derived from the inverse function of a conditional distribution function:

$q_{\alpha}\left(Y_{t} \mid \mathcal{F}_{t}\right)=F_{Y}^{-1}\left(\alpha \mid \mathcal{F}_{t}\right)$ 
where $F_{Y}\left(Y_{t} \mid \mathcal{F}_{t}\right)$ is the predicted conditional distribution function of $\mathrm{Y}_{\mathrm{t}}$. The inverse is to compute $q_{\alpha}\left(Y_{t} \mid \mathcal{F}_{t}\right)$ from

$\int_{-\infty}^{q_{\alpha}\left(Y_{t} \mid \mathcal{F}_{t}\right)} f \gamma\left(y \mid \mathcal{F}_{t}\right) d y=\alpha$

where $f \gamma\left(y \mid \mathcal{F}_{t}\right)$ is the predicted conditional distribution function. The quantile forecasting models $q_{\alpha}\left(Y_{t} \mid \mathcal{F}_{t}\right)$ are computed by solving the equation

$C_{u}\left(F_{X}\left(x_{t+1}\right), F_{Y}\left(q_{\alpha}\left(Y_{t} \mid \mathcal{F}_{t}\right)\right)=\alpha\right.$

To evaluate the predictive ability of those quantile forecasting models $q_{\alpha}\left(Y_{t} \mid \mathcal{F}_{t}\right)$ obtained from the seven $(I=7)$ copula functions for $\mathrm{C}(\mathrm{u} ; \mathrm{v})$, we use the "check" loss function of Koenker and Bassett $(1978)^{4}$. The expected check loss for a quantile forecast $q_{\alpha}\left(Y_{t} \mid \mathcal{F}_{t}\right)$ at a given $\alpha$ is

$Q(\alpha)=E\left[\alpha-I\left(Y_{t}-q_{\alpha}\left(Y_{t} \mid \mathcal{F}_{t}\right)<0\right)\right]\left(Y_{t}-q_{\alpha}\left(Y_{t} \mid \mathcal{F}_{t}\right)\right)$

We denote the $\mathrm{k}^{\text {th }}$ type of copula function as $\mathrm{C}_{\mathrm{k}}(\mathrm{u} ; \mathrm{v})(\mathrm{k}=1, \ldots, 1=7)$. For each copula distribution function $\mathrm{C}_{\mathrm{k}}(\mathrm{u} ; \mathrm{v})$, we also denote the corresponding quantile forecast as $q_{\alpha, k}\left(Y_{t} \mid \mathcal{F}_{t}\right)$ and its expected check loss as $\mathrm{Q}_{\mathrm{k}}(\alpha)$. To compare copula model 1 (benchmark) and model $\mathrm{k}(=$ $2, \ldots, 1)$, we consider the corresponding check loss-differential

$D_{k}=Q_{1}(\alpha)-Q_{k}(\alpha)$

We can estimate $D_{k}$ by

$\widehat{D}_{k, p}=\widehat{Q}_{1, p}(\alpha)-\widehat{Q}_{k, p}(\alpha)$

where

$$
\hat{Q}_{k, p}(\alpha)=\frac{1}{p} \sum_{t=R}^{T-1}\left[\alpha-I\left(Y_{t}-q_{\alpha}\left(Y_{t} \mid \mathcal{F}_{t}\right)<0\right)\right]\left(Y_{t}-q_{\alpha}\left(Y_{t} \mid \mathcal{F}_{t}\right)\right), \quad k=1, \ldots, l
$$

\footnotetext{
${ }^{4}$ For detailed information, please refer to Lee and Yang (2014).
} 
The conditional quantile forecasts from using the copula distribution function $\mathrm{C}_{\mathrm{k}}(\mathrm{k}=2, \ldots)$ with the largest value $\widehat{D}_{k, p}$ will be preferred.

\subsubsection{Directional predictability test}

We employ the recent directional predictability test of Han et al. (2016) to complement the GCD test because investors may want to use the GFSI to predict the movement of RBC; this follows the need to access the forecasting performance of RBC using the GFSI as a predictor. The null hypothesis is that the GFSI has no directional predictability for another time series. The added advantage of the cross-quantilogram of Han et al. (2016) over GCD is its ability to detect the magnitude, duration, and direction of the relationship between the GFSI and RBC spontaneously, whilst GCD failed to do so. Another advantage is that the model allows us to select arbitrary quantiles for both the GFSI and RBC, rather than pre-set quantiles for GCD. Furthermore, the use of the bootstrap technique allows for the use of large lags in the directional predictability test. The cross-quantilogram proposed by Han et al. (2016) can provide a quantile-to-quantile relationship from the GSFI to RBC. The linear quantile regression can be specified as

$q_{\alpha\left(\tau_{t+1} \mid \mathcal{F}_{t}\right)}=\beta_{0, \alpha}+\beta_{1, \alpha} x_{t}+\beta_{2, \alpha} x_{t} q_{\alpha}\left(\tau_{t} \mid \mathcal{F}_{t-1}\right)+\beta_{3, \alpha}\left|\tau_{t}\right|$

where $\tau_{t}$ and $x_{t}$ are the RBC and GSFI, respectively, and $q_{\alpha\left(\tau_{t+1} \mid \mathcal{F}_{t}\right)}$ is the conditional quantile of the RBC given the information $\mathcal{F}_{t}$ at time t. The cross-quantilogram $\hat{p}_{\alpha}(k)$ and the portmanteau tests $\hat{Q}_{\alpha}^{(p)}$ of the Box-Ljung versions are provided in the figures to detect the directional predictability from the RBC to GSFI. For the quantiles of $\operatorname{RBC} \mathrm{q}_{1}\left(\alpha_{1}\right)$, we consider a wide range for $\alpha_{1}=0.05,0.1,0.2,0.3,0.5,0.7,0.8,0.9$ and 0.95 . For the quantiles of GSFI $q_{2}\left(\alpha_{2}\right)$, we consider a wide range for $\alpha_{2}=0.1,0.5$, and 0.9. In each graph, we show the $95 \%$ bootstrap confidence intervals for no predictability based on 1000 bootstrapped replicates. The maximum lag we consider is 2 months (i.e., $\mathrm{k}=60$ ). To estimate the critical values from the limiting distribution, we could use the nonparametric estimation using the stationary bootstrap (SB) of Politis and Romano (1994). The SB is a block bootstrap method with blocks of random lengths. The SB resample is strictly stationary, conditional on the original sample. Alternatively, we can apply the self-normalized approach proposed in Lobato (2001) to test the absence of 
autocorrelation of a time series that is not necessarily independent. A key ingredient of the selfnormalized statistic is an estimate of cross-correlation based on subsamples.

Compared to the GCD, the directional predictability approach of Han et al. (2016) provides several added advantages. The first is related to its ability to detect the magnitude, duration, and direction of the relationship between the GFSI and RBC spontaneously, such that investors can use this information to inform their trading strategies. Second, the directional predictability test allows researchers to select arbitrary quantiles for the GFSI and RBC rather than pre-set quantiles, as in the case of GCD. Third, the use of the bootstrap technique allows for the use of large lags in the directional predictability test. The directional predictability test of Han et al. (2016) was used by Jiang et al. (2016) to investigate the daily, overnight, intraday, and rolling return spillovers of four key agricultural commodities-soybeans, wheat, corn, and sugarbetween the U.S. and Chinese futures markets. The authors found the empirical model very useful in capturing the extreme quantiles dependence between markets.

\section{Empirical Results}

\subsection{Results for marginal and copula models}

The probability density of the empirical copula is presented in Table 2 . We rank the pair of series in ascending order and then divide each series evenly into 10 bins. Bin 10 includes the observations with the highest values, and bin 1 includes observations with the lowest values. The ranks for the GFSI (i) are on the vertical axis, whilst the ranks for RBC (j) are on the horizontal axis. For the vertical axis, observations increase from the bottom to the top. For the horizontal axis, observations increase from left to right. Cell $(1,1)$ is located on the lower-left corner, cell $(10,1)$ is located on the lower-right corner, cell $(10,10)$ is located on the upper-right corner, and cell $(1,10)$ is located on the upper-left corner. The number of observations in cell $(1,1)$ reveals information about a positive left-tail dependence, the number of observations in cell $(1,10)$ reveals information about a negative right-tail dependence, the number of observations in cell $(10,10)$ reveals information about a positive right-tail dependence, and the number of observations in cell $(10,1)$ reveals information about a negative left-tail dependence between the two states. There are several noteworthy observations: cell $(1,1)$ has a low number, and there is no positive left-tail dependence (see lower-left corner, Table 2). When the global financial 
markets are generally healthy, it is unlikely that we will observe an extreme decrease in the Bitcoin market. Cell $(10,1)$ has a high number, and there is strong negative left-tail dependence (lower-right corner, Table 2). This finding indicates that the Bitcoin market is prosperous when the GFSI is very low. Cell $(1,10)$ has a high number, and there is strong negative right-tail dependence (upper-left corner, Table 2). Investors must rationally manage risks under this scenario, in which an extremely stressed financial market and a substantial decrease in the Bitcoin market occur simultaneously. An extreme joint loss is likely to be higher than a normal value-at-risk (VaR). Cell $(10,10)$ has a high number, and there is strong positive right-tail dependence (upper-right corner, Table 2). It is interesting to observe that the Bitcoin market can perform well when the global financial markets are in depression. This evidence justifies that Bitcoin provides a channel against global financial stress.

The ARMA(1,1)-tGARCH(1,1) model has been chosen for the marginal model as shown in Table 3. In the diagnostics section, $\mathrm{Q}(\mathrm{P})$ and $\mathrm{Q}^{2}(\mathrm{P})$ are $\mathrm{Q}$-statistics for testing the hypothesis of no serial correlation in the standardized residuals and squared standardized residuals, respectively. ARCH (P) is the LM test for the hypothesis of no autoregressive conditional heteroscedasticity in the standardized residuals. These statistics each have a chi-square distribution with $\mathrm{P}$ degrees of freedom. Based on the estimation results, the $\mathrm{t}$-stat is significant for all coefficients in the model for both the GFSI and RBC. The Q-statistics suggest no serial correlation in the standardized residuals of GSFI and RBC. The LM test suggests no heteroscedasticity in the standardized residuals of the GSFI and RBC.

Table 4 reports the estimated results for our data by applying nine candidate copulas, including normal, Clayton, Rotated Clayton, Plackett, Frank, Gumbel, Rotated Gumbel, Student's t and Symmetrised Joe-Clayton. In accordance with Patton (2006), we calculate the copula likelihood for each candidate copula. Based on the copula likelihood, we further calculate two information criteria: Akaike information criterion (AIC) and Bayesian information criterion (BIC). Additionally, we compute the tail dependence for each fitted copula. According to the copula log likelihood, the Gumbel copula provides the best fit for our data. According to AIC and BIC, the Gumbel copula is the best model for our data. Only through the Gumbel copula can we find tail 
dependence in the right tail. All other copulas indicate no tail dependence. Overall, the dependence structure of our data would be best captured by the Gumbel copula ${ }^{5}$.

\subsection{Results of the GCD test}

From subsection 4.1, we find evidence of a right tail dependence by the Gumbel copula, whilst all other copulas indicate no tail dependence. Although we identify the Gumbel copula as the appropriate copula for our data, the analysis provides no information about the causality between the GFSI and RBC. Therefore, this section seeks to provide a more informative test to examine the GCD as a tool to explore a causal relationship between the GSFI and the return of Bitcoin. The results of testing GCQ in p-values are reported in Table 5. The small p-values of the reality check signal the rejection of the null hypothesis, indicating that there is a copula function to model GCQ and produce a better quantile forecast of the RBC by conditioning on the GSFI. We can observe that a quantile forecasting model with no Granger causality in the quantile is rejected in many quantiles, except for the quantile at $40 \%, 50 \%$, and $60 \%$ with evidence at 1 percent significance level. This result shows that the GSFI strongly Granger-causes the RBC at the left tail (poor performance) and right tail (superior performance) but not at the centre (usual performance) of the distribution of the RBC conditional on the GSFI.

\subsection{Results of the directional predictability test}

In section 4.2, we examined the relationships between the GFSI and RBC at the extremes of the return distributions rather than only at the centre and confirmed the hypothesis that causality between the GFSI and RBC is only anticipated at high quantiles of the GFSI and RBC because $\mathrm{RBC}$ may act as a safe haven when the global financial market is in panic. However, investors may want to use the GFSI to predict the movement of the RBC, suggesting the need to conduct a complementary analysis to investigate directional predictability. To this end, we apply the directional predictability test of Han et al. (2016), and the results are presented in Figs. 1-3.

\footnotetext{
${ }^{5} \mathrm{We}$ also considered the possibility of the regime- dependent copula of Wang et al. (2013). However, the results are insignificant (for details, please see the appendix).
} 
Figs. 1(a) and 1(b) are for the case when the Bitcoin return is in the lower quantile, i.e., q2 $\left(\alpha_{2}\right)$ for $\alpha_{2}=0.1$. The cross-quantilogram $\hat{p}_{\alpha}(k)$ for $\alpha_{1}=0.2$ is positive and significant after the second week. This finding means that when GSFI is very low, there is less likely to be a very large negative loss for Bitcoin. The cross-quantilogram $\mathrm{p}_{-}^{\wedge} \alpha(\mathrm{k})$ for $\alpha_{1}=0.7,0.8,0.9$, and 0.95 is negative and significant for most lags, indicating that when GSFI is very low, it is less likely to have a very large positive gain for Bitcoin. Fig. 1(b) shows that the Box-Ljung test statistics are significant for quantiles $\alpha_{1}=0.1,0.2,0.3,0.5,0.8$ and 0.9 .

Figs. 2(a) and 2(b) are for the case in which the Bitcoin return is in the median, i.e., $\mathrm{q}_{2}\left(\alpha_{2}\right)$ for $\alpha_{2}=0.5$. If the distributions of Bitcoin returns and the predictor are symmetric, the median return forecast will be equal to the mean return forecast. For $\alpha_{1}=0.5$ and $\alpha_{2}=0.5$, the cross quantilograms are insignificant for nearly all lags. The cross-quantilogram $\hat{p}_{\alpha}(k)$ for $\alpha_{1}=0.2$ and 0.3 is positive and significant for all lags. This finding means that when the GSFI is lower than the median, it is less likely to result in a very large negative loss for Bitcoin. The crossquantilogram $\hat{p}_{\alpha}(k)$ for $\alpha_{1}=0.2$ and 0.3 is positive and significant for all lags. This finding means that when the GSFI is lower than the median, it is less likely to have a very large negative loss for Bitcoin. Fig. 2(b) shows the Box-Ljung test statistics for $\alpha_{2}=0.5$.

Figs. 3(a) and 3(b) are for the case in which Bitcoin return is in the higher quantile, i.e., q2 ( $\alpha 2)$ for $\alpha 2=0.9$. Compared to the previous case of $\alpha 2=0.5$, the cross quantilograms have very different trends in addition to much larger absolute values. For $\alpha 1<0.5$, the cross quantilograms are negative and significant for approximately 50-60 days. This finding implies that when financial stress is higher than the 0.9 quantile, there is an increased likelihood of having very large negative losses to Bitcoin for a maximum of 50-60 days. For $\alpha 1=0.8$ and 0.9 , the cross quantilograms are positive and significant for more than 60 days. This finding implies that when risk is very high (higher than the 0.8 quantile), there is an increased likelihood of having a very large positive gain for the next 60 days. Fig. 3(b) shows the Box-Ljung test statistics for $\alpha_{2}=0.9$. 
In summary, when global financial stress is in a higher quantile, in general, the absolute value of the cross-quantilogram is higher, and the cross-quantilogram is significantly different from zero for larger lags. Our results exhibit a more complete quantile-to-quantile relationship between financial stress and Bitcoin return and show how the relationship changes for different lags. This evidence on Bitcoin's safe-haven property against global financial stress in certain quantiles from a predictive perspective adds to that reported by Bouri et al. (2017b) in general and Bouri et al. (2017a) in particular. The evidence can be explained by the well-documented view that Bitcoin is part of an alternative economy (Bouri et al., 2017), and its price formation depends on certain non-economic and non-financial factors and Bitcoin attractiveness indicators (Ciaian and Rajcaniova, 2016) such as the anonymity of payment transactions (EBA, 2014), use in illegal activities (Böhme et al., 2015), and computer-programming enthusiasts (Yelowitz and Wilson, 2015).

\section{Conclusion}

Initially introduced as an electronic payment system equivalent to cash that could be used nearly anonymously in e-commerce, Bitcoin has quickly gained ground as an investment asset. A great deal of attention has been devoted to the technological, cryptographic, and legal aspects of Bitcoin. However, empirical evidence of its economic and financial aspects, particularly its role as a safe haven against global financial stress, is relatively scarce. This paper addressed this void by examining the quantile dependence between Bitcoin and global financial stress, which is measured by the GFSI, from July 18, 2010, to December 29, 2017. Interestingly, the GFSI captures global stress better than the VIX because it aggregates 23 measures of stress covering factors that reflect deteriorating economic fundamentals and poorly functioning financial systems. Methodologically, we considered the quantile dependence using copula functions, given the inability of conventional methods to appropriately capture the dependence between Bitcoin returns and the GFSI, as the bivariate joint distribution is not normally distributed. This paper not only found evidence of right-tail dependence but also computed the inverse of the conditional copula distribution function as a necessary step to obtain the conditional quantile functions and examine the Granger causality in different quantiles; in addition, it showed that global financial stress strongly Granger-causes Bitcoin returns at the left tail (deficient performance) and the right tail (superior performance) but not at the middle (average performance) of the joint 
distribution. Furthermore, we focused on the quantile-to-quantile relation from a predictive perspective and revealed evidence of directional predictability from the GFSI to Bitcoin returns, suggesting Bitcoin's ability to act as a safe-haven against global financial stress for approximately 60 days.

Overall, our findings support the literature on the valuable role of Bitcoin returns (Bouri et al., 2017a, 2017c; Brière et al., 2015; Dyhrberg, 2016a, 2016b; Ji et al., 2017) and extended it by showing the directional quantile dependence. This extension is important and useful to practitioners and policy-makers in an era of potentially high global financial stress. However, Bitcoin's possible benefits as a financial asset must be considered along with its associated volatility, which is documented in numerous studies (Pieters and Vivanco, 2017). It would also be premature to ensure that Bitcoin's role as a valuable investment will not be interrupted by a technological glitch. Future research can use the quantile dependence approach to more thoroughly examine Bitcoin's safe-haven property against conventional assets and commodities. 


\section{References}

Balcilar, M., Bouri, E., Gupta, R., Roubaud, D., 2017. Can volume predict Bitcoin returns and volatility? A quantiles-based approach. Econ. Model. 64, 74-81.

Böhme, R., Christin, N., Edelman, B., Moore, T., 2015. Bitcoin: economics, technology, and governance. J. Econ. Perspect. 29, 213-238.

Bollerslev, T., Wooldridge, J.M., 1992. Quasi-maximum likelihood estimation and inference in dynamic models with time-varying covariances. Econometric reviews, 11(2), 143-172.

Bouri, E., Azzi, G., Dyhrberg, A.H., 2017c. On the return-volatility relationship in the Bitcoin market around the price crash of 2013. Economics 11, 1-16.

Bouri, E., Gupta, R., Tiwari, A.K., Roubaud, D., 2017a. Does Bitcoin hedge global uncertainty? Evidence from wavelet-based quantile-in-quantile regressions. Financ. Res. Lett. 23, 8795.

Bouri, E., Molnár, P., Azzi, G., Roubaud, D., Hagfors, L.I., 2017b. On the hedge and safe haven properties of Bitcoin: is it really more than a diversifier? Financ. Res. Lett. 20, 192-198.

Brière, M., Oosterlinck, K., Szafarz, A., 2015. Virtual currency, tangible return: portfolio diversification with bitcoin. J. Asset Manag. 16, 365-373.

Ciaian, P., Rajcaniova, M., 2016. The digital agenda of virtual currencies: can Bitcoin become a global currency? Inf. Syst. e-Bus. Manag. 14, 883-919.

Dyhrberg, A.H., 2016a. Bitcoin, gold and the dollar - A GARCH volatility analysis. Finance Research Letters 16, 85-92.

Dyhrberg, A.H., 2016b. Hedging capabilities of bitcoin. Is it the virtual gold? Financ. Res. Lett. $16,139-144$.

Strunk Jr., W., White, E.B., 2000. The Elements of Style. Longman, New York.

Han, H., Linton, O., Oka, T., Whang, Y.-J., 2016. The cross-quantilogram: measuring quantile dependence and testing directional predictability between time series. J. Econom. 193, 251-270.

Hong, Y., Li, H., 2005. Nonparametric specification testing for continuous-time models with applications to term structure of interest rates. Rev. Financ. Stud. 18, 37-84. 
Ji, Q., Bouri, E., Gupta, R., Roubaud, D., 2017. Network Causality Structures among Bitcoin and other Financial Assets: A Directed Acyclic Graph Approach, Report No 201729. University of Pretoria, Department of Economics, Pretoria.

Jiang, H., Su, J.-J., Todorova, N., Roca, E., 2016. Spillovers and directional predictability with a cross-quantilogram analysis: the case of U.S. and Chinese agricultural futures. J. Futur. Mark. 36, 1231-1255.

Koenker, R., Bassett Jr, G., 1978. Regression quantiles. Econometrica: journal of the Econometric Society, 33-50.

Lee, T.-H., Yang, W., 2014. Granger-causality in quantiles between financial markets: using copula approach. Int. Rev. Financ. Anal. 33, 70-78.

Lobato, I.N., 2001. Testing that a dependent process is uncorrelated. J. Am. Stat. Assoc. 96, 1066-1076.

Luther, W.J., Salter, A.W., 2017. Bitcoin and the bailout. Q. Rev. Econ. Financ. 66, 50-56.

Nelsen, R.B., 1999. An Introduction to Copulas. Springer, New York.

Ning, C., 2010. Dependence structure between the equity market and the foreign exchange market-a copula approach. J. Int. Money Finance 29, 743-759.

Patton, A.J., 2006. Estimation of multivariate models for time series of possibly different lengths. J. Appl. Econ. 21, 147-173.

Pieters, G., Vivanco, S., 2017. Financial regulations and price inconsistencies across Bitcoin markets. Inf. Econ. Policy 39, 1-14.

Politis, D.N., Romano, J.P., 1994. The stationary bootstrap. J. Am. Stat. Assoc. 89, 1303-1313.

Popper, N., 2015. Digital Gold: The Untold Story of Bitcoin. Penguin Books Limited, London.

Wang, Y.-C., Wu, J.-L., Lai, Y.-H., 2013. A revisit to the dependence structure between the stock and foreign exchange markets: a dependence-switching copula approach. J. Bank. Financ. 37, 1706-1719.

Weber, B., 2014. Bitcoin and the legitimacy crisis of money. Cambridge J. Econ. 40, 17-41.

Yelowitz, A., Wilson, M., 2015. Characteristics of Bitcoin users: an analysis of Google search data. Appl. Econ. Lett. 22, 1030-1036. 

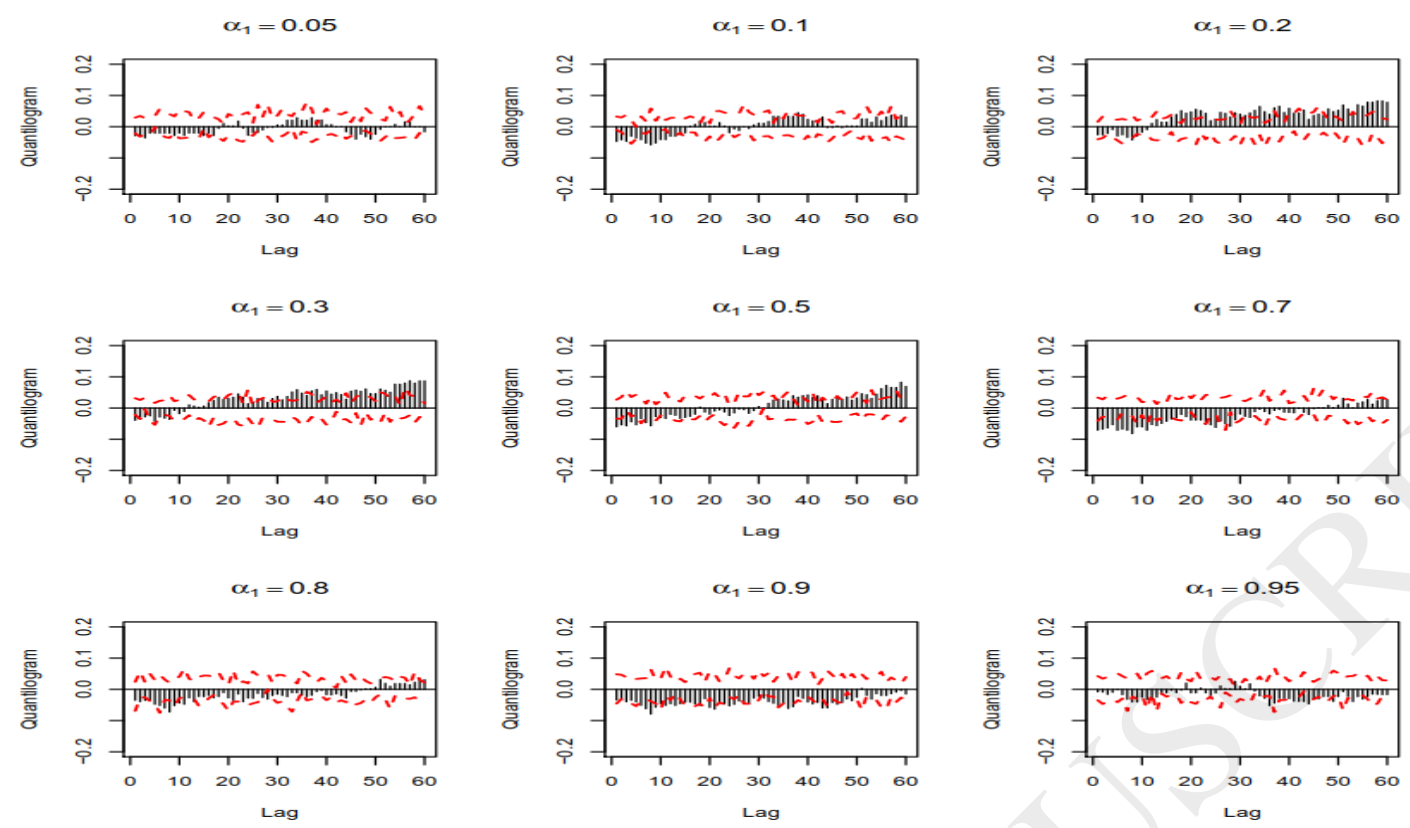

Fig. 1(a). The sample cross quantilogram $\hat{p}_{\alpha}(k)$ for $\alpha 2=0.1$ to detect directional predictability from the GSFI to RBC. Bar graphs describe sample cross quantilograms, and lines are the $95 \%$ bootstrap confidence intervals.
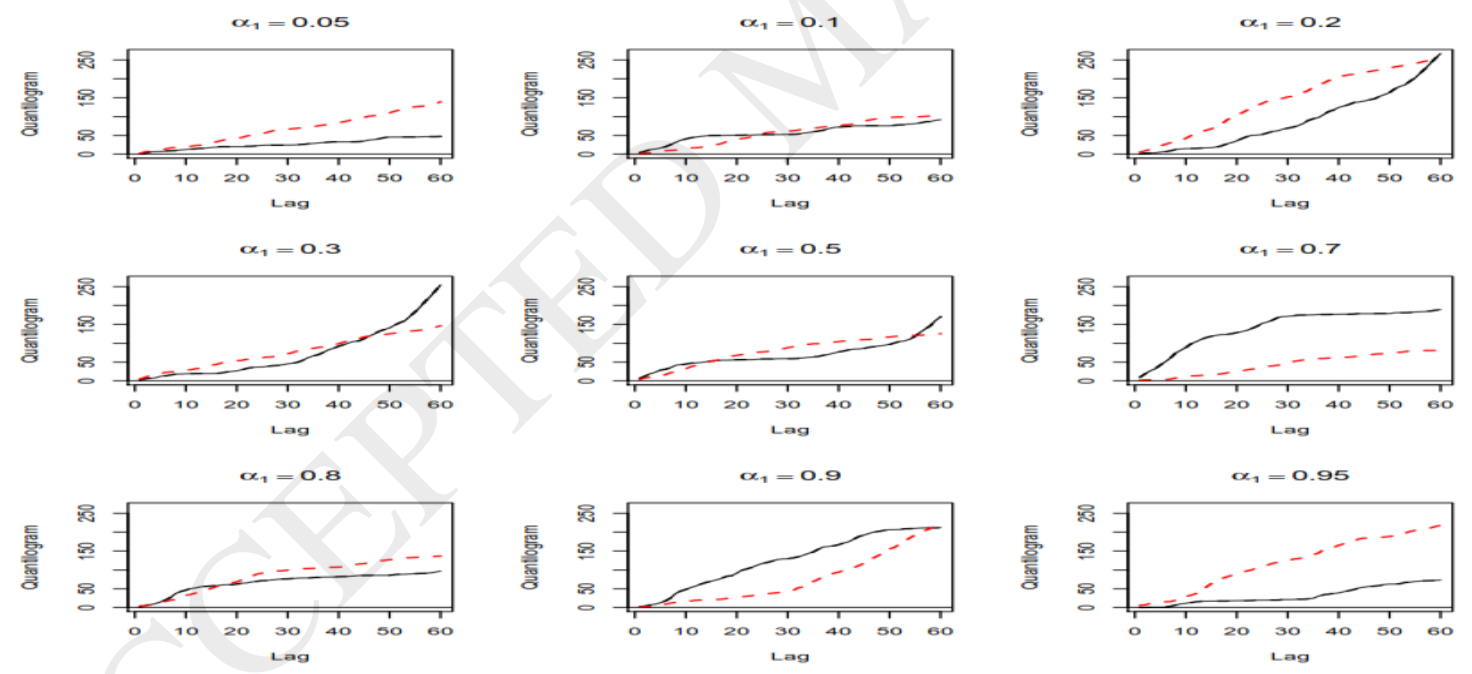

Fig. 1(b). Box-Ljung test statistic $\hat{Q}_{\alpha}^{(p)}$ for each lag p and quantile using $\hat{p}_{\alpha}(k)$, with $\alpha 2=0.1$. The dashed lines are the $95 \%$ bootstrap confidence intervals. 

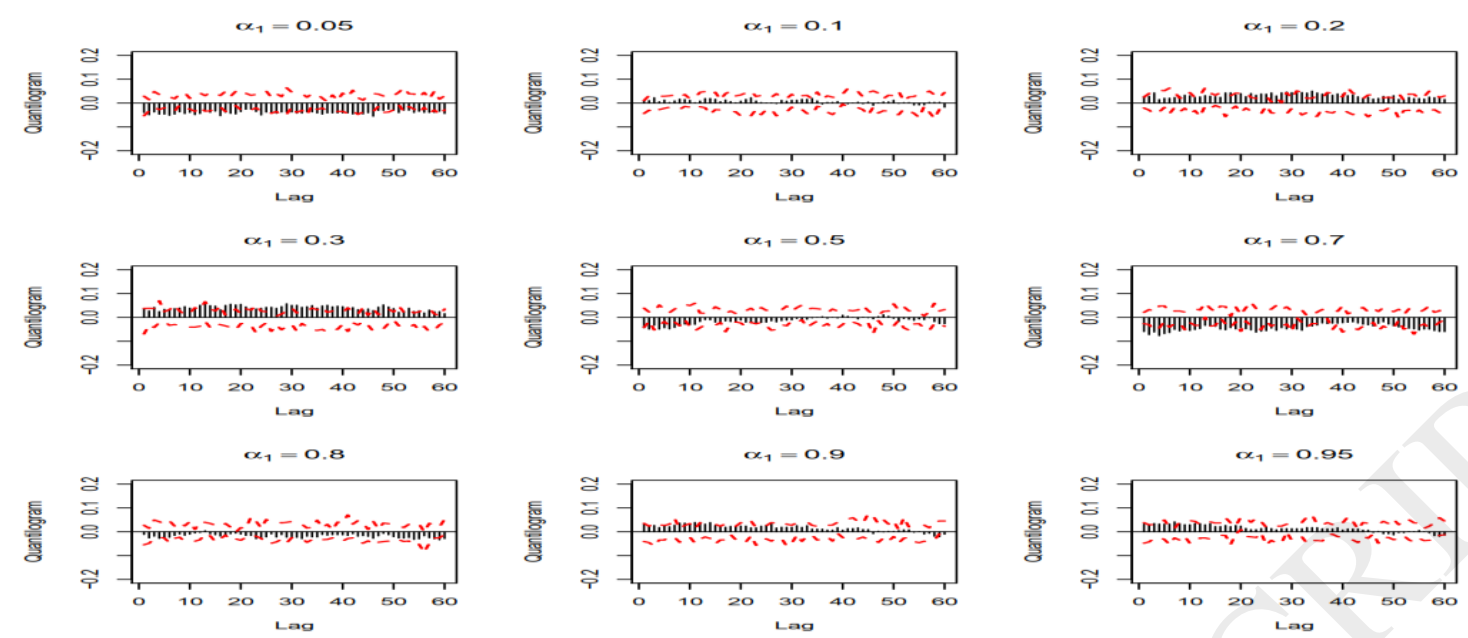

Fig. 2(a). The sample cross quantilogram $\hat{p}_{\alpha}(k)$ for $\alpha 2=0.5$ to detect directional predictability from the GSFI to RBC. Bar graphs describe sample cross quantilograms, and lines are the $95 \%$ bootstrap confidence intervals.
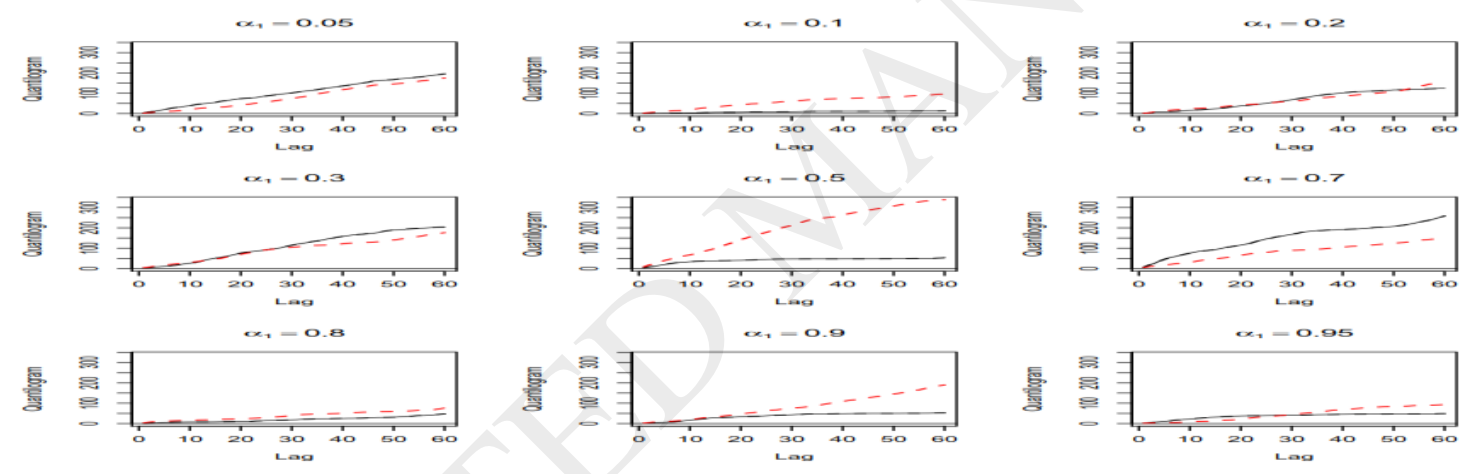

Fig. 2(b). Box-Ljung test statistic $\hat{Q}_{\alpha}^{(p)}$, for each lag $\mathrm{p}$ and quantile using $\hat{p}_{\alpha}(k)$ with $\alpha 2=0.5$. The dashed lines are the $95 \%$ bootstrap confidence intervals.

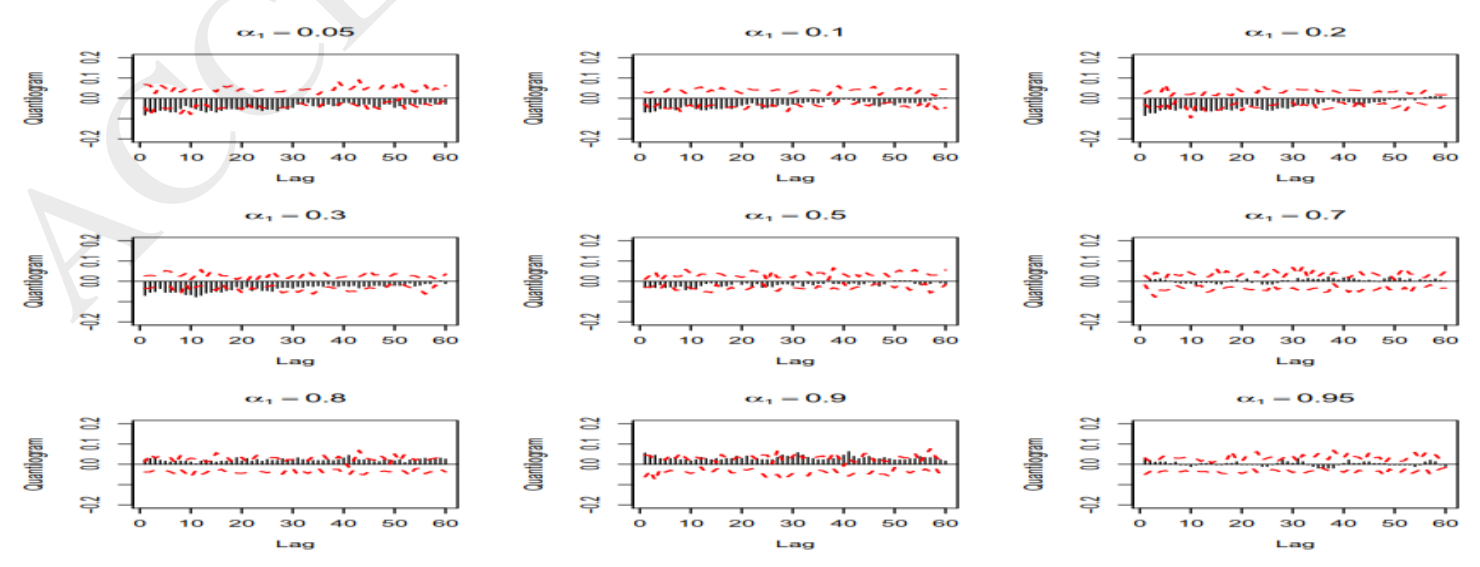


Fig. 3(a). The sample cross quantilogram $\hat{p}_{\alpha}(k)$ for $\alpha 2=0.9$ to detect directional predictability from the GSFI to RBC. Bar graphs describe sample cross quantilograms, and lines are the $95 \%$ bootstrap confidence intervals.
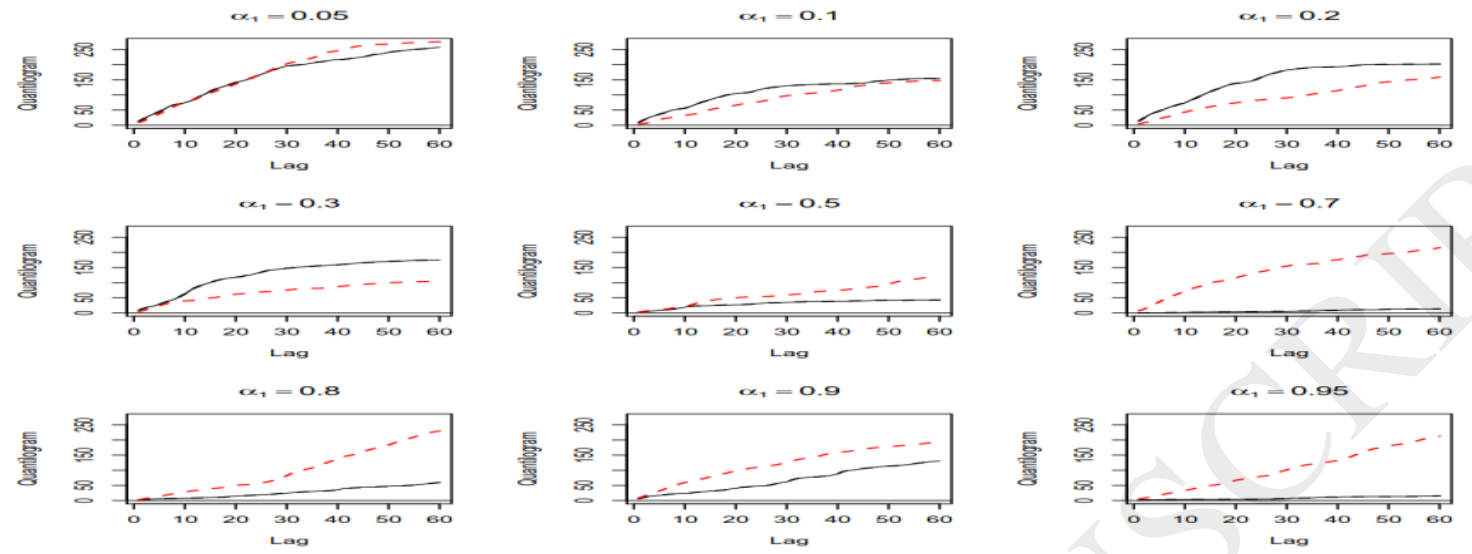

Fig. 3(b). Box-Ljung test statistic $\hat{Q}_{\alpha}^{(p)}$ for each lag p and quantile using $\hat{p}_{\alpha}(k)$, with $\alpha 2=0.9$. The dashed lines are the $95 \%$ bootstrap confidence intervals. 
Table 1. Summary statistics.

\begin{tabular}{lll}
\hline Series & GFSI & RBC \\
\hline Mean & 0.105 & 0.006 \\
Maximum & 1.310 & 0.500 \\
Minimum & -0.530 & -0.470 \\
Standard devaition & 0.355 & 0.065 \\
Skewnes & 0.784 & 0.016 \\
Kurtosis & 3.572 & 12.338 \\
Jarque-Bera test & 225.738 & 7062.835 \\
Unconditional Correlation & -0.053 & \\
\hline
\end{tabular}

Note: This table presents summary statistics of the GFSI and Bitcoin returns (RBC) for the period July 18, 2010, to December 29, 2017.

Table 2. Empirical copula for the GFSI and Bitcoin return.

\begin{tabular}{lllllllllll}
\hline & $\begin{array}{l}\text { Bitcoin- } \\
\text { lowest }\end{array}$ & & & & & & & & & $\begin{array}{l}\text { Bitcoin- } \\
\text { highest }\end{array}$ \\
GFSI-highest & 34 & 27 & 18 & 12 & 21 & 11 & 19 & 14 & 16 & 31 \\
& 13 & 15 & 18 & 28 & 52 & 27 & 19 & 10 & 14 & 6 \\
& 12 & 12 & 24 & 19 & 24 & 27 & 32 & 11 & 13 & 17 \\
& 15 & 20 & 15 & 17 & 21 & 16 & 21 & 19 & 22 & 21 \\
& 22 & 17 & 18 & 14 & 22 & 13 & 13 & 21 & 21 & 28 \\
& 21 & 21 & 22 & 15 & 17 & 21 & 20 & 29 & 19 & 11 \\
& 17 & 20 & 25 & 26 & 7 & 27 & 18 & 23 & 21 & 17 \\
& 19 & 26 & 23 & 21 & 12 & 18 & 20 & 24 & 22 & 24 \\
GFSI-lowest & 31 & 16 & 18 & 23 & 8 & 13 & 15 & 17 & 23 & 12 \\
\hline
\end{tabular}

Notes: The pair of returns are ranked in ascending order and then each series is divided evenly into 10 bins. Bin 10 includes the observations with the highest values, and bin 1 includes observations with the lowest values. The ranks for the return of Bitcoin (i) in the pair are on the vertical axis, whilst the ranks for the GFSI (j) in the pair are on the horizontal axis. For the vertical axis, returns increase from the bottom to the top. For the horizontal axis, returns increase from left to right. 
Table 3. Estimation of marginal models.

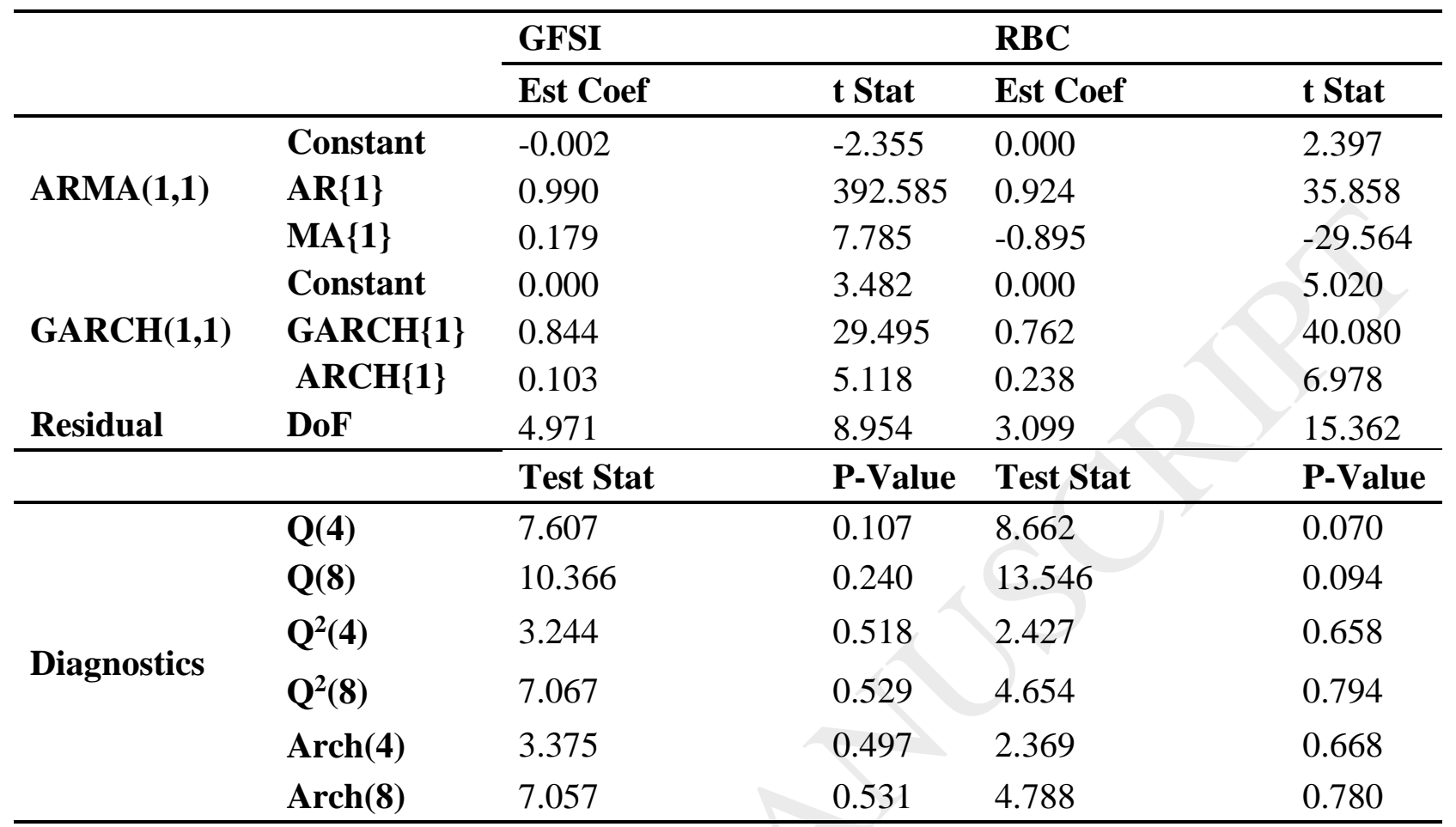

Notes: The order of ARMA is $(1,1)$, and the order of GARCH is $(1,1)$, which is sufficient for our data with evidence from the autocorrelation and heteroscedasticity tests. This model is parsimonious; it can capture both autocorrelation and heteroskedasticity.

Table 4. Copulas model estimation.

\begin{tabular}{lcllllll}
\hline Copula Type & \multicolumn{2}{c}{ Estimated Parameter } & $\begin{array}{l}\text { Copula Log } \\
\text { Likelihood }\end{array}$ & AIC & BIC & $\begin{array}{l}\text { Left-tail } \\
\text { Dependence }\end{array}$ & $\begin{array}{l}\text { Right-tail } \\
\text { Dependence }\end{array}$ \\
\hline Normal & $\rho$ & -0.009 & -0.074 & -0.147 & -0.144 & 0.000 & 0.000 \\
Clayton & $\theta$ & 0.000 & 0.005 & 0.011 & 0.014 & 0.000 & 0.000 \\
Rotated Clayton & $\theta$ & 0.011 & -0.110 & -0.218 & -0.215 & 0.000 & 0.000 \\
Plackett & $\theta$ & 0.999 & 0.000 & 0.001 & 0.004 & 0.000 & 0.000 \\
Frank & $\theta$ & 0.004 & 0.001 & 0.002 & 0.005 & 0.000 & 0.000 \\
Gumbel & $\theta$ & 1.008 & -0.250 & -0.499 & -0.496 & 0.000 & 0.010 \\
Rotated Gumbel & $\theta$ & 1.000 & 0.009 & 0.019 & 0.022 & 0.000 & 0.000 \\
Student's t & $\rho$ & -0.008 & -0.187 & -0.372 & -0.366 & 0.000 & 0.000 \\
Symmetrised & $v$ & 81.327 & & & & & \\
Joe-Clayton & upper tail & 0.000 & 1.177 & 2.355 & 2.361 & 0.000 & 0.000 \\
\hline \multicolumn{1}{r}{} & lower tail & 0.000 & & & & &
\end{tabular}

Notes: The normal copula has zero tail dependence. The Clayton copula has zero upper-tail dependence. The rotated Clayton copula has zero lower-tail dependence. The Plackett copula has zero tail dependence. The Frank copula has zero tail dependence. The Gumbel copula has zero lower-tail dependence. The rotated Gumbel copula has zero upper-tail dependence. The Student's t copula has symmetric tail dependence. The SJC copula parameters are the tail-dependence coefficients, but in reverse order. 
Table 5. Testing for GCQ.

\begin{tabular}{lllllllllllll}
\hline $1 \%$ & $5 \%$ & $10 \%$ & $20 \%$ & $30 \%$ & $40 \%$ & $50 \%$ & $60 \%$ & $70 \%$ & $80 \%$ & $90 \%$ & $95 \%$ & $99 \%$ \\
\hline 0 & 0 & 0 & 0 & 0 & 0.038 & 0.546 & 0.4 & 0 & 0 & 0 & 0 & 0 \\
\hline
\end{tabular}

Notes: We compute the quantile forecasts by inverting the parametric conditional copula distribution. We use six copulas (Gaussian, Frank, Clayton, Clayton Survival, Gumbel and Gumbel Survival copulas). The check loss functions are compared to evaluate the predictive ability of different quantile forecasting using different copula models. The benchmark quantile forecasts are computed using the independent copula such that there is no GCQ. Reported are the bootstrap p-values for testing the null hypothesis that none of these six copula models (which model GCQ) produces a better quantile forecast than the independent copula (which gives no GCQ). The small pvalues of the reality check indicate the rejection of the null hypothesis, indicating that there is a copula function to model GCQ and produce a better quantile forecast. 


\section{Appendix}

\section{Copula dependence model}

Sklar's Theorem. Let $F_{X Y}$ be a joint distribution function. $F_{X Y}$ has two marginal distributions $F_{X}$ and $F_{Y}$. There exists a copula $C$ such that all $x, y$ in $\boldsymbol{R}$,

$F_{X Y}=C\left(F_{X}(x), F_{Y}(y)\right)$

The copula $C$ is unique if marginal distributions $F_{X}$ and $F_{Y}$ are continuous; otherwise, $C$ is uniquely determined on $\operatorname{Ran}\left(F_{X}\right) \times \operatorname{Ran}\left(F_{Y}\right)$. Sklar's theorem enables us to model the marginal distributions and the dependence structure separately. Within our context, a bivariate joint cumulative distribution function of returns of Bitcoin and the GFSI can be decomposed into two marginal cumulative distribution functions and a copula cumulative distribution function.

Assuming $F_{X}$ and $F_{Y}$ are differentiable, the bivariate joint density is defined as

$f_{X Y}=\frac{\partial^{2} F_{X Y}}{\partial x \partial y}=f_{X}(x) f_{Y}(y) c(u, v)$

where $u$ and $v$ are the "probability integral transforms" of $x$ and $y$ based on $F_{X}(x)$ and $F_{Y}(y)$, respectively. $f_{X}(x)$ and $f_{Y}(y)$ are marginal densities of $x$ and $y$, respectively, and $c(u, v)=$ $\frac{\partial^{2} C(u, v)}{\partial u \partial v}$ is the copula density. Therefore, the bivariate joint density is expressed as the product of the two marginal densities and the copula density.

One particularly important dependence measure that copula can capture is the tail dependence, which measures the probability that two variables are jointly in their lower or upper tails. Tail dependence can be viewed as a pronounced spike in the data points in the plot of the lower-left or upper-right corner of a copula probability density. Intuitively, lower or upper-tail dependence is a relatively high probability density in the lower or upper quantile of the joint distribution. In Nelsen (1999), the left and right-tail dependence coefficients of a copula are defined as

$\varphi_{L}=\lim _{u \rightarrow 0} \mathbf{P}\left[Y \leq F_{Y}^{(-1)}(u) \mid X \leq F_{X}^{(-1)}(u)\right]=\lim _{u \rightarrow 0} \frac{C(u, u)}{u}$
$\varphi_{R}=\lim _{u \rightarrow 1} \mathbf{P}\left[Y>F_{Y}^{(-1)}(u) \mid X>F_{X}^{(-1)}(u)\right]=\lim _{u \rightarrow 1} \frac{1-2 u+C(u, u)}{1-u}$

where $C$ is a copula function. 


\section{Estimation methods and parametric copulas}

The quasi-maximum likelihood method (Bollerslev and Wooldridge, 1992) is applied to estimate the marginal models. The variance-covariance matrix of the estimated coefficients is adjusted accordingly. We use the Canonical Maximum Likelihood method to transform the standardized residuals, which are based on a cumulative distribution function (CDF) ${ }^{6}$, into uniform distribution. We present the parametric copulas used in the paper and their tail-dependence properties.

\section{Normal Copula}

$$
C_{N}(u, v)=\Phi_{\rho}\left(\Phi^{-1}(u), \Phi^{-1}(v)\right)
$$

where $\Phi^{-1}(\cdot)$ is the inverse of the distribution function of a standard normal random variable, and $\Phi_{\rho}(\cdot)$ denotes the distribution function of a bivariate normal random vector with zero means and correlation $\rho$. A normal copula has zero tail dependence.

\section{Clayton Copula}

$$
C_{C}(u, v)=\left[\max \left(u^{-\theta}+v^{-\theta}-1,0\right)\right]^{-\frac{1}{\theta}}, \text { where } \theta \in[-1, \infty] \backslash\{0\}
$$

The Clayton copula has zero upper-tail dependence but positive lower-tail dependence.

\section{Rotated Clayton Copula}

$$
C_{R C}(u, v)=\left[\max \left((1-u)^{-\theta}+(1-v)^{-\theta}-1,0\right)\right]^{-\frac{1}{\theta}} \text {, where } \theta \in[-1, \infty] \backslash\{0\}
$$

The rotated Clayton copula has zero lower-tail dependence but positive upper-tail dependence.

\section{Plackett Copula}

$$
C_{P}=\frac{1+(\theta-1)(u+v)-\sqrt{[1+(\theta-1)(u+v)]^{2}-4 \theta(\theta-1) u v}}{2(\theta-1)} \text {, where } \theta \in(0, \infty)
$$

The Plackett copula has zero tail dependence.

\section{Frank Copula}

$$
C_{F}(u, v)=-\frac{1}{\theta} \ln \left(1+\frac{\left(e^{-\theta u}-1\right)\left(e^{-\theta v}-1\right)}{e^{-\theta}-1}\right), \text { where } \theta \in(-\infty, \infty) \backslash\{0\}
$$

The Frank copula has zero tail dependence.

\section{Gumbel Copula}

$$
C_{G}(u, v)=\exp \left(-\left[(-\ln u)^{\theta}+(-\ln v)^{\theta}\right]^{\frac{1}{\theta}}\right), \text { where } \theta \in[1, \infty]
$$

The Gumbel copula has zero lower-tail dependence but positive upper-tail dependence.

\section{Rotated Gumbel Copula}

\footnotetext{
${ }^{6}$ The empirical marginal cumulative distribution function is computed by $\hat{F}_{k}(x)=\frac{1}{T+1} \sum_{t=1}^{T} \mathbf{I}\left(\eta_{k, t} \leq x\right)$, where $I(\cdot)$ is an indicator function.
} 


$$
C_{R G}(u, v)=\exp \left(-\left[(-\ln (1-u))^{\theta}+(-\ln (1-v))^{\theta}\right]^{\frac{1}{\theta}}\right) \text {, where } \theta \in[1, \infty]
$$

The rotated Gumbel copula has zero upper-tail dependence but positive lower-tail dependence.

\section{Student's t Copula}

$$
C_{S T}(u, v)=\Psi_{\rho}\left(\Psi^{-1}(u, d), \Psi^{-1}(v, d), d\right)
$$

where $\Psi_{\rho}(\cdot)$ is the distribution function of a bivariate Student's t distribution with correlation $\rho$ and degree of freedom parameter $d$, and $\Psi^{-1}$ is the inverse of the distribution function for the univariate Student's t distribution with zero means and degree of freedom $d$. The Student's $\mathrm{t}$ copula has symmetric tail dependence.

\section{Symmetrised Joe-Clayton Copula}

where

$$
C_{S J C}(u, v)=\frac{1}{2}\left(C_{J C}(u, v)+C_{J C}(1-u, 1-v)+u+v-1\right)
$$

$$
\begin{gathered}
C_{J C}(u, v)=1-\left(1-\left\{\left[1-(1-u)^{\kappa}\right]^{-\gamma}+\left[1-(1-v)^{\kappa}\right]^{-\gamma}-1\right\}^{-\frac{1}{\gamma}}\right)^{\frac{1}{\kappa}} \\
\kappa=\frac{1}{\log _{2}\left(2-\tau^{U}\right)} \\
\gamma=-\frac{1}{\log _{2}\left(\tau^{L}\right)}
\end{gathered}
$$

and $\left\{\tau^{U}, \tau^{L}\right\} \in(0,1)$

\section{Marginal and Copula models}

The ARMA(1,1)-tGARCH(1,1) model has been chosen for the marginal model. There are three motivations to use this model. First, our data contain a serial correlation. The level equation is chosen to be ARMA $(1,1)$. Second, our data have heteroscedasticity. The volatility equation is chosen to be GARCH $(1,1)$. Third, there are many outliers in the data. The standardized residuals follow the Student-t distribution. The order of ARMA is $(1,1)$ and the order of GARCH is $(1,1)$, which is sufficient for our data with evidence from the autocorrelation and heteroscedasticity tests. This model is parsimonious, which can capture both autocorrelation and heteroskedasticity ${ }^{7}$. The model is given by:

\footnotetext{
${ }^{7}$ When $k=1$, that is the model for the GFSI; when $k=2$, that is the model for the RBC.
} 


$$
\begin{aligned}
R_{k, t} & =\alpha_{k, 0}+\alpha_{k, 1} R_{k, t-1}+\alpha_{k, 2} \varepsilon_{k, t-1}+\varepsilon_{k, t} \quad k=1,2 \\
h_{k, t} & =\beta_{k, 0}+\beta_{k, 1} \varepsilon_{k, t-1}^{2}+\beta_{k, 2} h_{k, t-1} \\
\eta_{k, t} & =\frac{\varepsilon_{k, t}}{\sqrt{h_{k, t}}}, \quad \eta_{k, t} \mid \Omega_{t-1} \sim t\left(d_{k}\right)
\end{aligned}
$$

where $\eta_{k, t}$ is the standardized residuals, which follows the Student-t distribution with $d_{k}$ degrees of freedom. The ARMA $(1,1)-\operatorname{tgARCH}(1,1)$ model is estimated by the quasi-maximum likelihood method.

Specifying the correct marginal models is a key step. With incorrect marginal models, the estimated copula model is unable to capture the correct dependence structure of two series. The $Q$-statistic and the $Q^{2}$-statistic are applied to examine the hypothesis of no serial correlation in the estimated standardized residuals. The ARCH-LM statistic is employed to ensure that no heteroscedasticity is in the estimated standardized residuals.

\section{Regime-dependent Copula}

We further assume that the copula function is time-varying, depending on an unobservable state variable $S_{t}$.

$$
C_{S_{t}}= \begin{cases}C_{G}(u, v)=\exp \left(-\left[(-\ln u)^{\theta_{1}}+(-\ln v)^{\theta_{1}}\right]^{\frac{1}{\theta_{1}}}\right) & \text { if } S_{t}=1 \\ C_{R G}(u, v)=\exp \left(-\left[(-\ln (1-u))^{\theta_{0}}+(-\ln (1-v))^{\theta_{0}}\right]^{\frac{1}{\theta_{0}}}\right) & \text { if } S_{t}=0\end{cases}
$$

where $u$ and $v$ are the "probability integral transforms" of the GFSI and RBC. $\theta_{1}$ is the parameter for the Gumbel copula, and $\theta_{0}$ is the parameter for the rotated Gumbel copula.

The unobservable state variable $S_{t}$ follows a two-state Markov chain. $S_{t}$ transits between two states according to the transition probabilities.

$$
P=\left[\begin{array}{ll}
P_{11} & P_{10} \\
P_{01} & P_{00}
\end{array}\right]
$$

where $P_{i j}=P\left(S_{t}=j \mid S_{t}=i\right)$ for $\{i, j\} \in\{0,1\}$.

The bivariate density function of the above model is defined as

$$
f_{t}^{T V}(x, y)=\sum_{i=0}^{1} P\left(S_{t}=i\right) c_{i}(u, v) f_{X}(x) f_{Y}(y)
$$

where $c_{i}(u, v)$ is the copula under regime $i \in\{0,1\}$, i.e. $c_{1}(u, v)=\frac{\partial^{2} c_{G}(u, v)}{\partial u \partial v}$, and $c_{0}(u, v)=$ $\frac{\partial^{2} C_{R G}(u, v)}{\partial u \partial v}$. Notice that we assume the copula functions are different under different regimes; however, the two marginal densities are the same across different regimes. The model with 
dependence switching enables us to discuss the asymmetric tail dependence and the transition between the left-tail dependence regime and the right-tail dependence regime. The log likelihood of the model of a parameter set $\boldsymbol{\Theta}=\left\{\boldsymbol{\theta}_{c}, \boldsymbol{\theta}_{X}, \boldsymbol{\theta}_{Y}\right\}$ with a given data $\boldsymbol{D}$ is

$$
\boldsymbol{L}(\boldsymbol{\Theta})=\boldsymbol{L}_{c}\left(\boldsymbol{\theta}_{c}\right)+\boldsymbol{L}_{X}\left(\boldsymbol{\theta}_{X}\right)+\boldsymbol{L}_{Y}\left(\boldsymbol{\theta}_{Y}\right)
$$

where $\boldsymbol{L}_{c}\left(\boldsymbol{\theta}_{c}\right)$ is the $\log$ likelihood of the copula density; $\boldsymbol{L}_{X}\left(\boldsymbol{\theta}_{X}\right)$ and $\boldsymbol{L}_{Y}\left(\boldsymbol{\theta}_{Y}\right)$ are the $\log$ likelihood of the two marginal densities.

$$
\begin{aligned}
& \boldsymbol{L}_{c}\left(\boldsymbol{\theta}_{c}\right)=\log \left[\sum_{i=0}^{1} P\left(S_{t}=i\right) c_{i}(u, v)\right] \\
& \boldsymbol{L}_{X}\left(\boldsymbol{\theta}_{X}\right)=\log \left[f_{X}(x)\right] \\
& \boldsymbol{L}_{Y}\left(\boldsymbol{\theta}_{Y}\right)=\log \left[f_{Y}(y)\right]
\end{aligned}
$$

where $\boldsymbol{\theta}_{c}=\left\{P_{00}, P_{01}, P_{10}, P_{11}\right\}, \boldsymbol{\theta}_{X}$ and $\boldsymbol{\theta}_{Y}$ are the parameter set for the two marginal models, respectively. Because of the structure of the log likelihood function, its three components can be optimized independently. Table A1 reports the results for the regime-dependent copula model; the result shows that the regime-switching effect is absent for our dataset (see Table A1), as $P_{11}$ is exactly 1.000 with SD 0.000 . Hence, the regime-switching is not suitable in our study. The Gumbel will always be chosen, and the rotated Gumbel will never be chosen. This finding can also be confirmed by smooth probability. This result is not surprising because Table 4 shows that there is no left-tail dependence for whatever copula is used, and there is very weak right-tail dependence only when the Gumbel copula is used.

Table A1. Estimation of the dependence-switching copula model.

\begin{tabular}{llll}
\hline & & Value & SD \\
\hline Gumbel & $\theta_{1}$ & 1.008 & 0.011 \\
\hline Rotated Gumbel & $\theta_{2}$ & 1.108 & 0.230 \\
Regime & $P_{11}$ & 1.000 & 0.000 \\
Switching & $P_{22}$ & 0.956 & 0.102 \\
& Copula $L V$ & -0.378 & \\
& AIC & 0.775 & \\
\hline
\end{tabular}

Notes: $\theta_{i}$ is the shape parameter of the dependence-switching copula. "Copula LV" denotes the estimated log likelihood value for the copula function. $P_{11}$ and $P_{22}$ are two transition probabilities. SD denotes the standard deviation. 